\title{
Incidence of Pericardial Effusion in Patients with Advanced Non-Small Cell Lung Cancer Receiving Immunotherapy
}

\author{
Maria Laura Canale - Andrea Camerini (D) - Giancarlo Casolo • \\ Alessio Lilli · Irma Bisceglia · Iris Parrini - Chiara Lestuzzi • \\ Jacopo Del Meglio · Cheti Puccetti · Lara Camerini · Domenico Amoroso • \\ Nicola Maurea
}

Received: April 15, 2020 / Published online: May 20, 2020

(C) The Author(s) 2020

\section{ABSTRACT}

Introduction: Cardiovascular toxicity of immunotherapy represents an underreported but potentially fatal side effect. A relatively high incidence of pericardial disease has been noticed in patients with non-small cell lung cancer (NSCLC).

Methods: We retrospectively analyzed a population of patients with advanced NSCLC receiving immune checkpoint inhibitors (ICIs)

Digital Features To view digital features for this article go to https://doi.org/10.6084/m9.figshare.12264863.

M. L. Canale · G. Casolo · A. Lilli · J. Del Meglio Cardiology Division, Azienda USL Toscana NordOvest, Versilia Hospital, Lido di Camaiore (LU), Italy

A. Camerini $(\bowtie) \cdot$ C. Puccetti · D. Amoroso Medical Oncology, Azienda USL Toscana NordOvest, Versilia Hospital, Lido di Camaiore (LU), Italy e-mail: andreacamerini@katamail.com

I. Bisceglia

Servizi Cardiologici Integrati, Azienda Ospedaliera

San Camillo-Forlanini, Rome, Italy

I. Parrini

Divisione di Cardiologia, Ospedale Mauriziano,

Torino, Italy

C. Lestuzzi

Cardiology Unit, Oncology Department, CRO

National Cancer Institute, Aviano (PN), Italy looking for the presence of pericardial effusion at baseline or during treatment. The study population was compared with a control group treated with chemotherapy. All patients were checked for the presence of concomitant pleural effusion.

Results: We identify 60 patients (36 male/24 female, median age 70 years [range 43-81]). Prevalent histology was adenocarcinoma (65\%) followed by squamous cell carcinoma (28\%) and large cell or not otherwise specified (NOS) carcinoma $(7 \%)$. Treatment consisted of nivolumab $3 \mathrm{mg} / \mathrm{kg}$ every 14 days (52 cases; 45 as second-line and 7 as third-line treatment) or

\section{Puccetti}

Clinical Research Task Force, Azienda USL Toscana Nord-Ovest, Versilia Hospital, Lido di Camaiore (LU), Italy

\section{Camerini}

School-Job Project, Liceo Scientifico Ulisse Dini, Pisa, Italy

N. Maurea

S.C. Cardiologia, Istituto Nazionale Tumori, IRCCS Fondazione G. Pascale, Napoli, Italy 
pembrolizumab $200 \mathrm{mg}$ (8 cases; all first-line treatment) for a total of 302 cycles delivered. Four out of 60 patients $(6.7 \%)$ developed pericardial effusion during treatment, in two cases (3.3\%) without concomitant pleural effusion, compared to 2 out of $60(3.3 \%)$ in the control group in one case without concomitant pleural effusion (1.6\%). Median time of onset was 40 days. Myocarditis was not observed.

Conclusion: Our findings confirm pericardial effusion as a relatively frequent side effect of immunotherapy in NSCLC. Clinicians should be aware of this specific toxicity in patients with metastatic NSCLC receiving immunotherapy and refer to a cardiologist for a multidisciplinary approach.

Keywords: Cardiac toxicity; Immune checkpoint inhibitors; Immunotherapy; Non-small cell lung cancer; Pericardial effusion

\section{Key Summary Points}

Why carry out this study?

Cardiac toxicity of immunotherapy is infrequent but potentially fatal.

Pericardial disease has been associated with immunotherapy specifically in patients with advanced non-small cell lung cancer (NSCLC) on the basis of retrospective pharmacovigilance analysis.

The association between pericardial effusion and immunotherapy in advanced NSCLC should be confirmed by real-world data.

\section{What was learned from the study?}

We observed a higher incidence of pericardial effusion in a real-world population of patients with advanced NSCLC receiving immunotherapy.

Pericardial effusion is a relatively frequent toxicity of immunotherapy in NSCLC and these patients should be referred to a cardio-oncology team.

\section{INTRODUCTION}

Immune checkpoint inhibitors (ICIs) represent to date the standard treatment for multiple advanced cancers, having proved a substantial survival benefit in various cancer types [1-4]. Compared to classic anticancer agents, ICIs might produce a wide spectrum of specific immune-related adverse events (IRAEs) affecting virtually all organs and systems $[5,6]$. Cardiovascular toxicity of ICIs is a rare but potentially fatal side effect [7]. Originally reported with an overall incidence ranging from $0.09 \%$ to $0.27 \%$ [8], more recent reports indicate a frequency slightly above $1 \%$ [9] with a poor outcome in a relatively high percentage of cases [10]. Recently, a wide retrospective pharmacovigilance analysis showed that ICI treatment was associated with higher reporting of myocarditis, pericardial diseases, and vasculitis. Interestingly, authors reported an association between cancer site and specific cardiovascular IRAEs. Pericardial diseases were, indeed, more frequent in patients with lung cancer, whereas myocarditis and vasculitis were more often found in patients with melanoma [11]. Altan et al. reported a case series of three patients with advanced non-small cell lung cancer (NSCLC) treated with ICIs who developed pericarditis, which was in two cases fatal [12]. Moreover, cardiovascular toxicity of immunotherapy could be associated with other IRAEs in different organs [13]. The real incidence of immunerelated pericarditis in patients with advanced NSCLC in daily clinical practice is unknown and on these grounds we conducted a retrospective analysis to check for the presence of pericardial effusion during ICIs treatment. To reduce the probability of confusing immunerelated pericardial effusion with local progression of lung cancer we also checked for the presence of concomitant pleural effusion. Finally, we explored the contemporary occurrence of other IRAEs, when a pericardial effusion was found. For hypothetical purposes only, the study population was compared with an age- and sex-matched control group of patients with advanced NSCLC treated with 
conventional chemotherapy to highlight, if present, a difference in incidence.

\section{METHODS}

We performed a database search of medical oncology files from 2017 to 2018 . We selected medical records of patients (over 18 years of age) with stage IIIB/IV NSCLC treated with an ICI, both anti-programmed cell death protein 1 (PD-1) or its ligand (PD-L1), outside clinical trials. The investigation conforms with the principles outlined in the Declaration of Helsinki. The protocol did not require formal approval by local ethical committee given the retrospective and observational design of the study. Patients gave their written consent to data collection and analysis for medical research and related publications. Patients' names were coded and not revealed. All available clinical and pathological characteristics were recorded with special attention to other concomitant IRAEs. Additional eligibility criteria (other than advanced NSCLC and ICIs treatment) were availability of baseline imaging of thorax, no pre-treatment pericardial effusion, availability of at least one disease reassessment imaging at thoracic level, a time span between baseline and first disease revaluation of at least 8 weeks. CT scan of the thorax (preferred) and chest X-ray were considered as adequate imaging for both cancer and pericardial effusion evaluations. The same imaging method had to be available both at baseline and during followup.

Survival data were collected from medical records or the registry office. Given the observational design of this retrospective analysis no formal statistical design was set up. Descriptive data are presented as percentages, while time data are presented as median with range. The control group was built by randomly choosing patients with advanced NSCLC treated in the same time period in our hospital with conventional chemotherapy and tested for significance with respect to the experimental group in terms of age, sex, and treatment line. Non-parametric Mann-Whitney test was used to compare continuous variable while categorical variables were evaluated by chi-square analysis or Fisher's exact test where appropriate. The control group presented an overlapping median age, a similar sex distribution, and similar allocation on treatment lines.

\section{RESULTS}

A total of 60 patients fulfilled the inclusion criteria and were included for analysis. For the vast majority of patients (57 out of 60 ) CT scans of thorax were available both at baseline and during follow-up. The study population was mainly composed of men (36 out of $60,60 \%$ ), patients with stage IV (54 out of $60,90 \%)$ NSCLC, and the median age was 70 (range 43-81) years. Prevalent histology was adenocarcinoma (39 out of 60,65\%) followed by squamous cell carcinoma (17 out of $60,28 \%$ ) and large cell or not otherwise specified (NOS) carcinoma (4 out of $60,7 \%$ ) (Table 1). All patients were treated according to the

Table 1 Study population characteristics $(n=60$, total delivered cycles 302)

\begin{tabular}{ll}
\hline Age (years) median (range) & $70(43-81)$ \\
Sex $(\mathrm{F} / \mathrm{M})$ & $36 / 24$ \\
Cancer stage $(n / \%)$ & \\
IIIB & $6 / 60(10 \%)$ \\
IV & $54 / 60(90 \%)$ \\
Histology $(n / \%)$ & \\
Adenocarcinoma & $39 / 60(65 \%)$ \\
Squamous cell carcinoma & $17 / 60(28 \%)$ \\
Large cell carcinoma/NOS & $4 / 60(7 \%)$ \\
Treatment $(n / \%)$ & \\
Nivolumab & $52 / 60(86.7 \%)$ \\
2nd line & $45 / 52(86.5 \%)$ \\
3rd line & $7 / 52(13.5 \%)$ \\
Pembrolizumab* & $8 / 60(13.3 \%)$ \\
\hline
\end{tabular}

NOS not otherwise specified

*All first-line treatment for high $(\geq 50 \%)$ PD-L1 tumor expression 
oncologists' judgement based on internal and international guidelines of scientific societies.

Fifty-two patients received nivolumab $3 \mathrm{mg} /$ $\mathrm{kg}$ I.V. every 14 days up until disease progression, patient refusal, or unacceptable toxicity; 45 out of these 52 as second-line and 7 out of 52 as third-line treatment. Nivolumab treatment was not limited by PD-L1 expression levels. The remaining eight patients received pembrolizumab $200 \mathrm{mg}$ flat dose I.V. every 21 days up until disease progression, patient refusal, or unacceptable toxicity; all of them as first-line treatment in relation to high (at least 50\%) tumor PD-L1 expression (Table 1). A total of 302 cycles were delivered. The control group patients received a platinum doublet as first-line treatment in eight cases, docetaxel (21 patients), orally administered metronomic vinorelbine (18 patients), and gemcitabine (six cases) as second-line treatment, and gemcitabine (five patients) and orally administered metronomic vinorelbine (two patients) as thirdline therapy.

In the whole study population, 4 out of 60 patients $(6.7 \%)$ developed pericardial effusion during ICIs treatment, in three patients during nivolumab and in one case during pembrolizumab treatment. Pericardial effusion was found in both adenocarcinoma (two cases), squamous cell carcinoma (one case), and NOS carcinoma (one case). Concomitant pleural effusion was observed in two out of these four cases (one unilateral and one bilateral); therefore, pericardial effusion in those was more likely to be related to lung cancer disease progression. To confirm this, the case with bilateral pleural effusion underwent right pleural drainage with positive cytology. The incidence of pericardial effusion only was 3.3\%. In the control group we observed two patients (3.3\%) developing pericardial effusion during chemotherapy, in one case with concomitant pleural effusion leading to an overall incidence of $1.6 \%$ ( 1 out of 60 ). The difference of incidence of pericardial effusion alone in the two groups $(3.3 \%$ vs $1.6 \%)$ was not significant because of the small sample size. Median time of onset of pericardial effusion in ICI-treated patients was 40 days from treatment start. Sole pericardial effusion in the only patient treated with chemotherapy was observed after 65 days. We did not observe any other IRAEs in patients developing pericardial effusion. In particular, myocarditis was not reported. Myocarditis was ruled out by a troponin level within the normal range coupled with normal echocardiography findings (normal cardiac function with no evidence of left ventricle dysfunction or abnormalities in wall motion score index) and no specific symptoms (fever, chest pain). Serum polymerase chain reaction assays were not performed to test for viral infections. Treatment with ICIs was temporarily stopped in the four cases developing pericardial/pleural effusion. In spite of the presence of pericardial/pleural effusion, all patients did not report any cardiac symptom related to that and they remained hemodynamically stable. All patients with evidence of pericardial effusion underwent echocardiography that showed no sign of cardiac tamponade. No patients underwent pericardial drainage. Echocardiography did not show any sign of pericardial invasion by lung cancer. Moreover, no symptoms related to pericarditis were reported by patients. In three out of four patients, treatment was permanently stopped because of disease progression outside the pericardium and general worsening of conditions (two cases with concomitant pleural effusion) and patient refusal (one case with pericardial effusion alone). In one case treatment was restarted given the persisting absence of symptoms related to pericardial effusion with no further toxicity.

\section{DISCUSSION}

Immune checkpoint inhibitor-based immunotherapy demonstrated an impressive clinical efficacy in the fight against several cancers with various compounds currently approved for the treatment of many forms of malignant tumors [1-4]. There is a widespread consensus that this therapy should now be considered as a cornerstone in anticancer treatment [14]. Together with its proven efficacy, the safety profile of anti-PD-1/PD-L1 is characterized by a lower percentage of classic chemotherapy side effects, making ICIs a valid 
and appealing therapeutic option [15]. Nevertheless, clinicians faced new types of toxicities associated with ICIs treatment related to the triggering of an excessive autoimmune response [16]. The so-called IRAEs might affect virtually all organs and systems $[5,6]$, including the cardiovascular system [7-10]. One possible explanation for heart involvement in excessive ICI-related autoimmune response may be the presence of PD-1 and PD-L1 proteins on cardiomyocytes, and animal studies have demonstrated that the cytotoxic Tlymphocyteassociated protein 4 (CTLA-4) and PD-1 deletion can cause autoimmune myocarditis [17-19]. From a clinical point of view, cardiovascular toxicities of immunotherapy, even though rare, may be severe with an unexpectedly high rate of fatal complications $[7,8,12]$. The real incidence of such toxicity is still uncertain and a possible underestimation should be taken into account. This could be caused by several factors: (a) missing information on this particular side effect from pivotal clinical trials; (b) a still limited clinical experience; (c) little attention of clinicians to cardiovascular symptoms that could be confused with disease progression, mainly in patients with lung cancer that represent the majority of cancer population treated with ICIs; (d) the clinical presentation of cardiac IRAEs could be overlooked. Even the large pharmacovigilance analysis recently published [11] could be biased by the low attention and reluctancy of clinicians to side effect reporting.

Our results confirmed the observation by Salem et al. [11] on a specific association between pericardial disease and lung cancer in patients treated with ICIs and indicate a possible real incidence slightly higher than expected. We observed a total of four cases $(6.7 \%)$ of pericardial effusion during anti PD-1 (nivolumab and pembrolizumab) treatment. Even after correction for contemporary pleural effusion (possibly related to disease progression), the final incidence was 3.3\% (Table 2). The observation of a sex- and age-matched advanced NSCLC control group receiving chemotherapy showed an overall incidence of pericardial effusion of $3.3 \%$ (two patients) reduced to $1.6 \%$ (one patient) after exclusion of one patient with contemporary pleural effusion (Table 2).
Table 2 Incidence and median onset time of pericardiac effusion in patients treated with ICIs $(n=60$, total delivered cycles 302) compared to a sex- and age-matched control group ( $n=60$, total delivered cycles 288 ) receiving chemotherapy

\begin{tabular}{lll}
\hline & ICIs & Chemotherapy \\
\hline $\begin{array}{l}\text { Overall incidence }(n / \\
\%)\end{array}$ & $4 / 60(6.7 \%)$ & $2 / 60(3.3 \%)$ \\
$\begin{array}{l}\text { Adjusted incidence }{ }^{\mathrm{a}} \\
(n / \%)\end{array}$ & $2 / 60(3.3 \%)$ & $1 / 60(1.6 \%)$ \\
$\begin{array}{l}\text { Onset time (median } \\
[\text { range }])\end{array}$ & 40 & 65 \\
\hline $\begin{array}{l}\text { a Excluding patients with contemporary occurrence of } \\
\text { pleural effusion } \\
\text { b Based on a single event }\end{array}$
\end{tabular}

Interestingly, no case of myocardial disease was observed, supporting the previously reported specific relation between advanced lung cancer and pericardial-only ICI-associated disease. All our patients developing pericardial effusion did not report specific symptoms, so pericardial effusion was an incidental finding on a scheduled CT scan for disease re-evaluation. This observation agrees with the report of mild cardiac toxicity of ICIs [20]. The (lucky) event of a non-clinically relevant cardiac toxicity of immunotherapy further complicates the scenario making it hard to determine the real incidence of cardiac IRAEs. A specific timing of cardiac IRAEs has been observed. Nearly all reports indicate cardiac toxicity as an early event during treatment [7-10]. Furthermore, we observed a median onset time of 40 days while in the comparison group the patient developed it later after 65 days (Table 2).

Study limitations include bias in the determination of the control group as it was not based on a random allocation; so, as previously stated, it should be considered for hypothetical purposes only. Moreover, the retrospective analysis and the single-institution data source may limit the clinical value of observed phenomena. 


\section{CONCLUSIONS}

The number of patients with cancer receiving an ICI treatment will rapidly increase in the near future in relation to the approval of new drugs and/or the extension of indications for those already available. The observation of a small but relevant occurrence of pericardial disease (possibly paired with myocarditis) specifically in patients with advanced NSCLC should be taken into account by the clinicians taking care of them. Proper cardiac evaluation strategies during treatment, mainly focusing on the initial period of therapy, should be a part of a defined follow-up tailor-made path. Our data may provide the background for a prospective, multicenter evaluation of incidence and management of cardiac side effects of ICIs in patients with NSCLC.

\section{ACKNOWLEDGEMENTS}

Funding. No funding or sponsorship was received for this study or for the publication of this article. No support grant has been provided for the present study.

Authorship. All named authors meet the International Committee of Medical Journal Editors (ICMJE) criteria for authorship for this article, take responsibility for the integrity of the work as a whole, and have given their approval for this version to be published.

Disclosures. All authors (Maria Laura Canale, Andrea Camerini, Giancarlo Casolo, Alessio Lilli, Irma Bisceglia, Iris Parrini, Chiara Lestuzzi, Jacopo Del Meglio, Cheti Puccetti , Lara Camerini, Domenico Amoroso and Nicola Maurea) have nothing to disclose relating to the present study.

Compliance with Ethics Guidelines. The investigation conforms with the principles outlined in the Declaration of Helsinki. The selection did not require formal approval by local ethical committee given the retrospective and observational design of the study. Patients gave their written consent to data collection and analysis for medical research and related publications. Patients' names were coded and not revealed.

Data Availability. The dataset of the study is not publicly available because this was not included in the patients' informed consent form.

Open Access. This article is licensed under a Creative Commons Attribution-NonCommercial 4.0 International License, which permits any non-commercial use, sharing, adaptation, distribution and reproduction in any medium or format, as long as you give appropriate credit to the original author(s) and the source, provide a link to the Creative Commons licence, and indicate if changes were made. The images or other third party material in this article are included in the article's Creative Commons licence, unless indicated otherwise in a credit line to the material. If material is not included in the article's Creative Commons licence and your intended use is not permitted by statutory regulation or exceeds the permitted use, you will need to obtain permission directly from the copyright holder. To view a copy of this licence, visit http://creativecommons.org/licenses/bync/4.0/.

\section{REFERENCES}

1. Rotte A. Combination of CTLA-4 and PD-1 blockers for treatment of cancer. J Exp Clin Cancer Res. 2019;38:255.

2. Ackermann CJ, Reck M, Paz-Ares L, Barlesi F, Califano R. First-line immune checkpoint blockade for advanced non-small-cell lung cancer: travelling at the speed of light. Lung Cancer. 2019;134:245-53.

3. Weiss SA, Wolchok JD, Sznol M. Immunotherapy of melanoma: facts and hopes. Clin Cancer Res. 2019;25:5191-201.

4. Chang AJ, Zhao L, Zhu Z, et al. The past, present and future of immunotherapy for metastatic renal cell carcinoma. Anticancer Res. 2019;39:2683-7.

5. Spain L, Walls G, Julve M, et al. Neurotoxicity from immune-checkpoint inhibition in the treatment of 
melanoma: a single centre experience and review of the literature. Ann Oncol. 2017;28:377-85.

6. Hofmann L, Forschner A, Loquai C, et al. Cutaneous, gastrointestinal, hepatic, endocrine, and renal side-effects of anti-PD-1 therapy. Eur J Cancer. 2016;60:190-209.

7. Varricchi G, Galdiero MR, Marone G, et al. Cardiotoxicity of immune checkpoint inhibitors. ESMO Open. 2017;2:e00247.

8. Johnson DB, Balko JM, Compton ML, et al. Fulminant myocarditis with combination immune checkpoint blockade. N Engl J Med. 2016;375: 1749-55.

9. Mahmood SS, Fradley MG, Cohen JV, et al. Myocarditis in patients treated with immune checkpoint inhibitors. J Am Coll Cardiol. 2018;71: 1755-64.

10. $\mathrm{Hu} \mathrm{JR}$, Florido R, Lipson EJ, et al. Cardiovascular toxicities associated with immune checkpoint inhibitors. Cardiovasc Res. 2019;115:854-68.

11. Salem JE, Manouchehri A, Moey M, et al. Cardiovascular toxicities associated with immune checkpoint inhibitors: an observational, retrospective, pharmacovigilance study. Lancet Oncol. 2018;19: 1579-89.

12. Altan $\mathrm{M}$, Toki $\mathrm{MI}$, Gettinger $\mathrm{SN}$, et al. Immune checkpoint inhibitor-associated pericarditis. J Thorac Oncol. 2019;14:1102-8.
13. Heinzerling L, Ott PA, Hodi FS, et al. Cardiotoxicity associated with CTLA4 and PD1 blocking immunotherapy. J Immunother Cancer. 2016;4:50.

14. Farkona S, Diamandis EP, Blasutig IM. Cancer immunotherapy: the beginning of the end of cancer? BMC Med. 2016;14:73.

15. Haanen JBAG, Carbonnel F, Robert C, et al. Management of toxicities from immunotherapy: ESMO Clinical Practice Guidelines for diagnosis, treatment and follow-up. Ann Oncol. 2017;28: iv19-iv42.

16. De Velasco G, Je Y, Bossé D, et al. Comprehensive meta-analysis of key immune-related adverse events from CTLA-4 and PD-1/PD-L1 inhibitors in cancer patients. Cancer Immunol Res. 2017;5:312-8.

17. Nishimura H, Okazaki T, Tanaka Y, et al. Autoimmune dilated cardiomyopathy in PD-1 receptordeficient mice. Science. 2001;291:319-22.

18. Okazaki T, Tanaka Y, Nishio R, et al. Autoantibodies against cardiac troponin I are responsible for dilated cardiomyopathy in PD-1-deficient mice. Nat Med. 2003;9:1477-83.

19 Baban B, Liu JY, Qin X, Weintraub NL, Mozaffari MS. Upregulation of programmed death-1 and its ligand in cardiac injury models: interaction with GADD153. PLoS One. 2015;10:e0124059.

20 Norwood TG, Westbrook BC, Johnson DB, et al. Smoldering myocarditis following immune checkpoint blockade. J Immunother Cancer. 2017;5:91. 